\title{
Cognitive Chrono-Ethnography (CCE): a methodology for anticipating future user needs
}

\author{
Muneo Kitajima ${ }^{\mathrm{a}^{*}}$ \\ ${ }^{a}$ Center for Service Research, National Institute of Advanced Industrial Science and Technology (AIST), 1-1-1 \\ Umezono, Tsukuba, Ibaraki, 305-8568, Japan
}

\begin{abstract}
This paper proposes Cognitive Chrono-Ethnography (CCE), a new study methodology for understanding people's in situ behavior selections in daily life. People select their next behavior to maximize their satisfaction for a given behavioral needs. They appropriately coordinate available cognitive resources to make the best decisions by using their knowledge of past experiences and by processing input from the environment and individual intrinsic state. When a study field is specified, CCE starts by defining critical parameters for understanding people's behavior by considering the nature of behavior selection processes in the field in question, and then designing ethnographical field observations by taking into account the fact that their results will be described in terms of the specified critical parameters. The participants' behavior is recorded, followed by a series of structured retrospective interviews for the purpose of describing their present behavior and obtaining their history of behavioral development. Analysis of the interview results aid in developing models of present behavior selections and their chronological changes in the past. These models serve as defining future needs of persons who follow the same developing paths with a certain amount of delay, e.g., a few years of delay. This paper describes a CCE study of spectators of professional baseball games at a ballpark who have become frequent visitors to a baseball stadium in 5 years.
\end{abstract}

Keywords: behavior selection, field observation, retrospective interview, Cognitive Chrono-Ethnography (CCE)

${ }^{*}$ Corresponding author. E-mail: kitajima.muneo@aist.go.jp 


\section{Introduction}

A person has moment-by-moment desire for spending satisfactory and happy times in his/her living environment. Each person has his/her own unique living history. The personal history is stored in his/her long-term memory and significantly influences his/her way of reacting to his/her environment with the given desire, i.e., decision making. What he/she wants to achieve at present can be considered as his/her present needs, $D(t-0)$, and what he/she will consider he/she wants to achieve in the future given the present needs are satisfied,

$$
D(t=0) \pi i v e n \text { D }(0)=\text { true }
$$

can be considered as his/her future needs.

Suppose a person, $\mathbf{A}$, whose desire state at present, $t=\mathbf{b}$, is equal to the desire state of another person, $\mathbf{B}$, in the past, $t=-\tau \in 0$, i.e.,

$$
D_{i}(t=0 \text { present })=D_{1}\left(r=\tau, \tau_{1} \text { past }\right)
$$

and this also applies to A's history up to the past time,

$$
\tau=\sigma(\theta \leq i<b)
$$

when $\mathbf{A}$ began to pay attention to the object toward which his/her desire is oriented now, and if the environment can be considered stable compared with the characteristic time of the change in the state of a person who interacts with this environment, it is likely that the person $\mathbf{A}$ will take the same development path as the person $\mathbf{B}$ has taken. Therefore,

$$
D(r)-D_{k}(\mathrm{t} \cdot \mathrm{T}) \mathrm{tor}-\sigma \leq \mathrm{t} \leq \mathrm{t} \text {. }
$$

In other words, $\mathbf{B}$ is $\boldsymbol{\tau}$ years ahead of $\mathbf{A}$ in terms of the change of desire state. In this scheme, anticipation of future user needs of A during the period spanning from the present, $i=\emptyset$, to the future time, $=\bar{\tau}$, reduces to revealing the history of desire state of $\mathbf{B}$ from the past time, $i=(\sigma \cdot \tau)$, to the present, $i=0$.

I have developed a study methodology that stems on this principle. The methodology consists of two studies. The first is to understand how a person interacts with the current environment with a given desire. The second is to understand his/her history of interaction with the environment with concerning the desire in question.

This methodology is called "Cognitive ChronoEthnography (CCE)." It deals with a person's moment-by-moment decision-making process, a cognitive process, in the real environment for the purpose of understanding chronological development of his/her interaction with the environment for him/her to achieve his/her desire. The study will be conducted in the real-word setting, and therefore, it is an ethnographical study. The methodology is for understanding people's in situ daily behavior selections.

\section{Requirements for the CCE methodology to study people's in situ behavior}

\subsection{What to understand}

I suggest that understanding human beings' in situ daily behavior selections involves understanding relationships between memes that were active at the time the behavior was undertaken and overtly observed behavior, by considering those factors as Two Minds [1,2], the multiplicity of goals, and the nature of memory processes.

Therefore, a CCE study must answer the following questions about behavioral events:

- Which memes were activated?

- Under which conditions were the memes activated?

- How had the memes been formed?

The answers will be analyzed to construct models that explain and predict people's behavior in the study field.

\subsection{How to understand}

What kinds of data are available for deriving answers to the above questions? The origins of the data are the results of observing people's daily behavior selection processes in real-world settings.

Data that are obtainable with little interference with the participants' activities are as follows.

- Behavior observation records: Investigators record the participants' behavior without intervening in their activities.

- Behavior measurement records: Sensors are attached to the participants to record their physiological activities (e.g., a pin microphone to record their vocalization, a small ear-mounted camera to record the scene they are viewing, and an electrocardiograph to record their physiological responses to the events).

- On-site self-reports: Study participants themselves take photos, brief notes, and voice recording concerning their activities while their memories of the events remain fresh.

- Retrospective interviews: Behavioral observation records, behavioral measurement records, and on-site self-reports described above are used to reconstruct participants' active memes at the time of events by conducting a series of retrospective interviews. 


\section{CCE procedure}

CCE is carried out in the following six steps.

- Step 1. Define the study field: It is important to specify the study field sufficiently. Manifestations of behavior-selection shaping factors under the characteristic atmosphere of the study field are observed in the study field.

- Step 2. Define critical parameters: Critical parameters are initial hypotheses about the behavior-selection shaping factors that should work when people's activities are organized in the study field. Steps 1 and 2 are con-ducted interchangeably to define the parameter space to be explored.

- Step 3. Select elite monitors: Study participants (elite monitors) are selected. Each point in the parameter space has values. The study question is "what such-and-such people would do in such-and-such way in such-and-such circumstance." Therefore, elite monitors are selected by consulting the parameter space. In this process, it is necessary that the points in the parameter space, which correspond to the elite monitors, are appropriate for analyzing the structure and dynamics of the study field.

- Step 4. Record the monitors' behavior: The elite monitors are expected to be-have as they normally do in the study field. Their behavior is recorded in such a way that the collected data is rich enough to consider the results in terms of the parameter space.

- Step 5. Conduct interviews: The collected data are used to clarify the structure of the meme of the elite monitors by conducting a series of structured interviews. The results are analyzed for the purpose of defining the basis of the representations of the collected data.

- Step 6. Construct models: The last step of CCE is to construct models that ad-dress "what such-and-such people would do in such-andsuch way in such-and-such circumstances."

\section{A case study of CCE: Why do fans repeat visits to the ballpark?}

This section describes a case study of CCE [5]. The field of study was the ballpark of a Japanese professional baseball team, the Hokkaido NipponHam Fighters. This study focused on the repeat visiting behavior of loyal fans of the Fighters. The specif- ic study questions were: "Why do loyal fans repetitively visit Sapporo Dome to watch professional baseball games?" and "How have they evolved to their current status of loyal fans?"

CCE has been applied to a variety of domains. Examples of CCE studies can be found in Kitajima \& Toyota (in press) [4] for passengers who try to find their way at unfamiliar train stations by utilizing signs, Kitajima, Tahira, and Takahashi \& Midorikawa (in press) [3] for visitors at a hot spring resort in Japan.

\subsection{Outline of the study}

\subsubsection{About the Hokkaido Nippon-Ham Fighters}

The Hokkaido Nippon-Ham Fighters are a professional baseball team in Japan's Pacific League. The team takes its name from the major shareholding company, Nippon Ham, which is the corporate name of Nippon Meat Packers, Inc. In 2004 the Fighters moved from Tokyo to Sapporo, the largest city on the island of Hokkaido. The team uses Sapporo Dome, a stadium located in Toyohira-ku, Sapporo, Hokkaido, Japan, that is primarily used for football and baseball. It is the home field of the football club Consadole Sapporo and the baseball team Hokkaido NipponHam Fighters. Sapporo Dome opened in 2001 and currently has 42,126 seats. This stadium hosted three games during the 2002 FIFA World Cup.

\subsubsection{Structures of fans (Figure 1).}

Loyal fans, or repeaters, of a professional baseball team have their own individual histories in arriving at their current fan stage. They started in a pre-fan stage, passed through the fan stage, and ultimately reached their current loyal-fan stage. In the pre-fan stage, fans know little about the team, or at most they pay a certain amount of attention to the team and/or have some interest in the team. However, their attitude toward the team is passive, and they exert no aggressive action.

Starting from this pre-fan stage, they advance to the fan stage, when they aggressively desire to have a relationship with the team. For example, fan-stage individuals display emotion towards the results of the games, and start to become interested in watching live games at the stadium. However, they do not have much interest in information about the team.

A fan-stage person advances to a loyal fan by breaking through these passive characteristics. Loyalstage fans aggressively collect information about the team, go to the stadium to watch live games when 


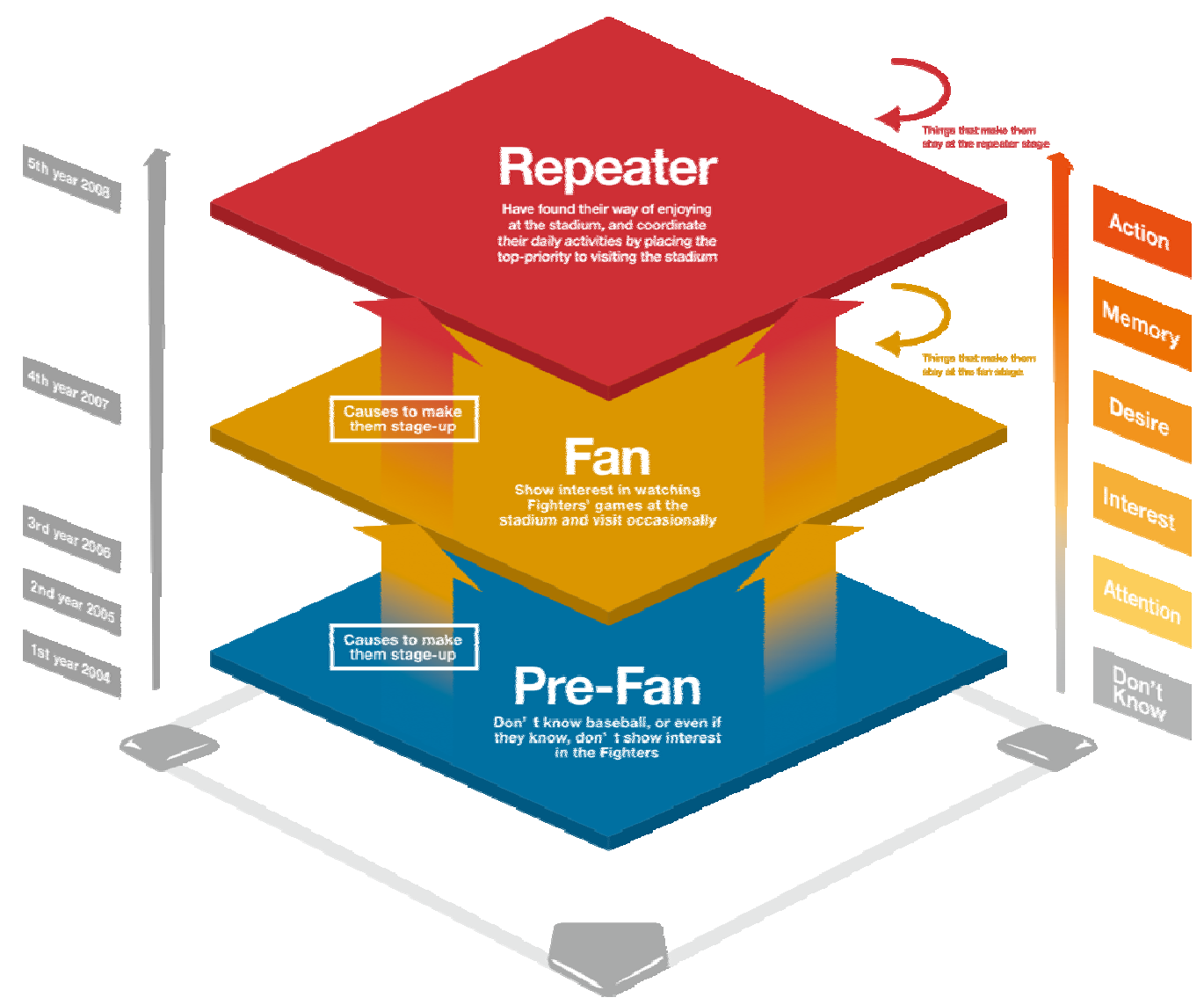

Fig.1: Structure of fans.

time allows, or even arrange their activities so as to give top priority to watching live games at the stadium.

\subsubsection{Study questions.}

During the five years since the Fighters moved to Sapporo, its number of fans has increased dramatically. There were 38,776 fans registered with the official fan club in $2004,41,817$ in 2005, 41,193 in 2006, 60,216 in 2007, and 74,974 in 2008 (as of September 30 of each year). It is easy to enumerate plausible reasons behind the continuous increase in the number of fans.

First is the so-called Shinjo effect. Outfielder Tsuyoshi Shinjo joined the Fighters in 2004, after his three-year career with Major League Baseball teams, the New York Mets and the San Francisco Giants. The phenomenon, known as the Shingo effect, was created with his outstanding talent in making professional baseball an entertaining show. Second, the Fighters won the Pacific League championship in the 2006 and 2007 regular seasons. Third is the contribu- tion of manager Hilman. Finally, we can list the efforts of the players and the front-office staff who visited various places, including local schools, in an effort to establish intimate relationships with local communities.

However, nobody knows exactly why the Fighters have achieved such great success. This long-standing lack of knowledge in the service industry hinders its productivity. Therefore, the Japanese government and the Ministry of Economy, Trade, and Industry have decided to fund research and development projects to help service providers implement services more efficiently by understanding successful practices based on scientific and technological underpinnings. For the Fighters, scientific underpinnings include understanding why fans repeatedly come to Sapporo Dome to watch the Fighters' games and how they enjoy them, and correlating such understanding with the events that the Fighters provided. 


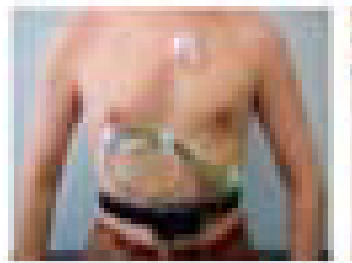

(a)

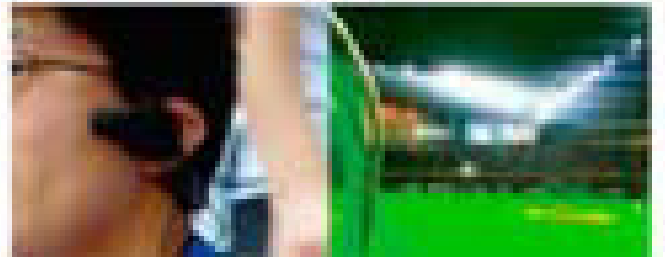

(b)

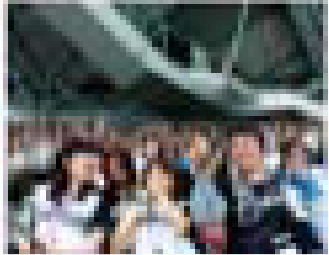

(d)

Fig. 2: Illustration of field observation. (a) an electrocardiograph and an accelerometer, (b) ear-mounted CCD camera, (c) the view of the ear-mount camera, and (d) three elite monitors in their seats watching a game

\subsection{A CCE study for understanding why spectators repeat professional baseball games}

This project was started at the beginning of the 2008 regular season for the purpose of establishing a set of hypotheses concerning the processes of developing repeaters who attend games hosted by the Hokkaido Nippon-Ham Fighters at Sapporo Dome.

We took the following approach to develop a set of hypotheses concerning the developmental processes of repeaters.

\subsubsection{Selection of monitors (CCE's step 3)}

We conducted a Web survey and recruited nine highly loyal fans (elite monitors) from the Fighters' fan club members who had different attitudes towards professional baseball, cheering, and merchandising, and had visited Sapporo Dome several times since the Fighters moved to Sapporo. The nine selected fans were supposed to represent different "fan styles" and had different histories in reaching their current fan status.

\subsubsection{Field observation (CCE's step 4)}

We had the elite monitors visit Sapporo Dome three times to watch designated Fighters-hosted games. We recorded their viewing behavior using a DVD camera recorder located three rows in front of the monitors' seats to capture their game-viewing behavior, installing a small ear-mounted CCD camera to record the scene they were viewing, recording their vocalizations with a pin microphone, and using an electrocardiograph and an accelerometer to capture their physiological responses to the events of the game (Figure 2). The designated games were a threegame series with the Softbank Hawks in July, a three-game series with the Orix Buffalos in August, and a three-game series with the Rakuten Golden Eagles in September. Each elite monitor was asked to attend all three series.

\subsubsection{Retrospective interviews (CCE's step 5)}

We conducted structured interviews after each visit to Sapporo Dome, replaying the behavior records, the viewing-scene records, and the broadcasted TV video of the game for the characteristic events, including scoring scenes, field events between innings, and events for which the participants exhibited remarkable changes in physiological data. Each participant was interviewed three times. The purpose of the first interview was to understand how the participants enjoy the game. The purpose of the second interview was to understand how participants developed their loyalty from the pre-fan stage several years ago, to the fan stage a few years ago, and then to the current repeating stage. The purpose of the third interview was to understand what triggered the state changes and what factors helped them retain each fan stage.

\subsubsection{Construct fan loyalty evolution model (CCE's step 6)}

We compiled the results of interviews in the form of a fan-loyalty evolution diagram (FLE diagram) that represented in detail how individual participants had evolved their loyalty by specifying triggers for stage changes, circumstances that made them stay at a particular stage, and activities in both the regular season and in the off-season. Nine FLE diagrams were created. We then collapsed them to derive models of developmental processes of repeaters, which will be described in the next section.

\subsection{Results: Developmental processes of repeaters}


The following section describes results of analysis of the evidence collected during the interview sessions that focused on triggers that caused monitors to step up a stage (i.e., from pre-fan stage to fan stage, and from fan stage to loyal-fan stage), and the conditions that made or make them stay in a particular stage [6]. These triggers and conditions define a rough qualitative model of the developmental process of fan loyalty.

\subsubsection{Progressing from the pre-fan stage to the fan stage}

Three common triggers were found in the study for advancing the elite monitors from the pre-fan stage to the fan stage. who had little knowledge about professional baseball and another three participants who had knowledge about professional baseball but did not have enough interest in it to progress to the fan stage. One event was an announcement by the then-star player, outfielder Tsuyoshi Shinjo, that he was retiring, relatively early in the regular season. This news was reported frequently in various media. The other event was that the Fighters were in the first championship race of the league and Japan's professional baseball leagues.

2) "Watch the fans cheering." - Two participants who had little knowledge about professional baseball and one participant who had little in-

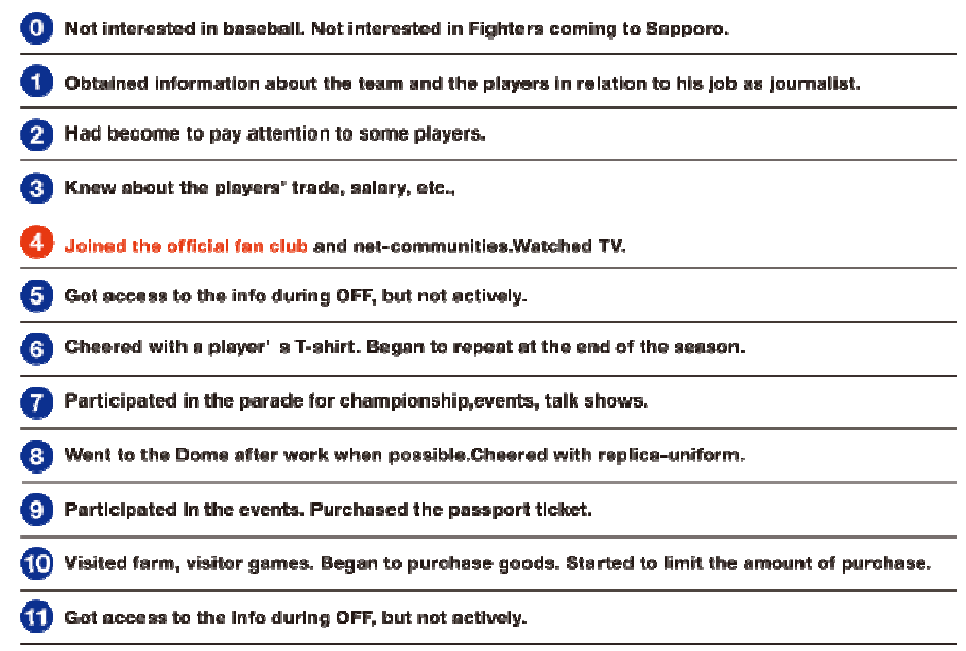

Fig. 3: Fan loyalty evolution diagram and characteristic episodes.

1) "Retirement of a star player" and "expectation of league championship." - In the 2006 regular season, two events triggered three participants terest in professional baseball advanced to the fan stage after watching live cheering in the stadium. 


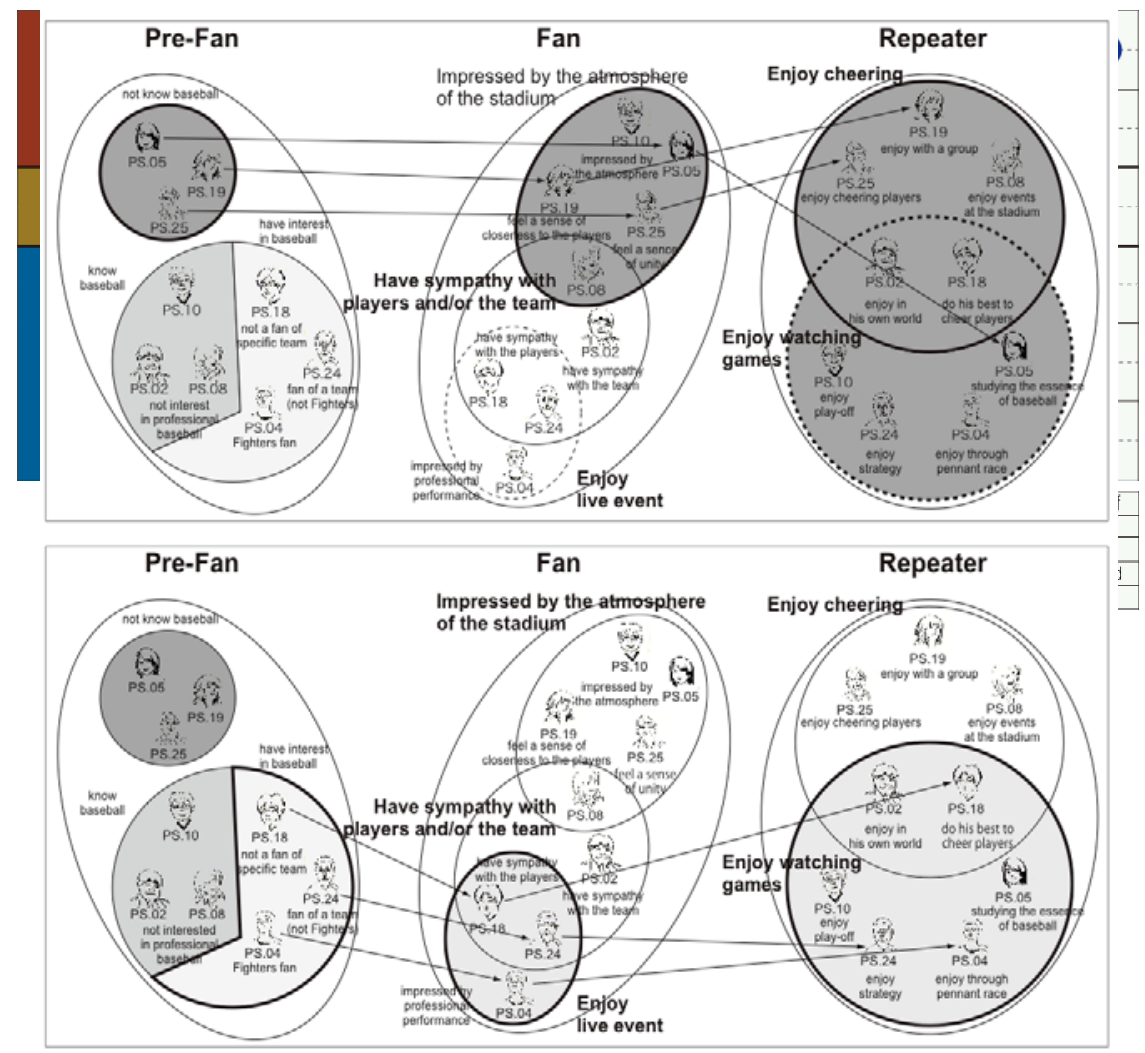

Fig. 4: The development processes from the pre-fan stage to the repeater stage. Top - pre-fans who did not know baseball well. Bottom - pre-fans who had interest in baseball but not had strong interest in watching games at the stadium.

3) "Know the players and the team" and "unexpected talent of players outside baseball." Regardless of their knowledge level of professional baseball, knowing players and the team triggered participants to progress to the fan stage. Three participants who knew professional baseball reacted to the players' behavior outside baseball, causing them to advance to the fan stage.

\subsubsection{Advancing from the fan stage to the loyal-fan stage}

Ten common triggers were found in the study for advancing the elite monitors from the fan stage to the loyal-fan stage.

1) "Watching live games at the stadium."

2) "Knowing the rules of baseball and the team."

3) "Watching games by oneself," "one's wife became a fan by following his lead," "communication with his/her friends at the stadium," or "meeting persons who visited the stadium." The common feature of these triggers is the establishment of an environment where fans could comfortably watch the games at the sta- dium with someone who contributed to building a relationship with them (e.g., spouse or friends).

4) "Presence of players who always come to mind." - Participants who had little knowledge about baseball or professional baseball, those who were fans of other professional baseball teams, and those who became fans at the end of the regular seasons tended to find opportunities that should provide information about players, teams, and the Fighters in particular. These participants were eager to attend off-season events such as talk shows and advanced to loyal fans in the next regular season.

5) "Collecting the Fighters' goods."

6) "Recording events of live games" and/or "collecting the recordings as proof of watching the games."

7) "Expectation of the climax series and the Nippon series," and "eagerness to watch those series."

8) "Communication with the other fans when watching live games." 
9) "Network community" that they accessed during live games to exchange information and post opinions.

10) "Seeing the players closely (e.g., visiting camp in Okinawa," and those who had special interest (or who followed pro-baseball) said that their greatest interest was in seeing live action on a professional field.

\subsubsection{Developing from a pre-fan to a repeater}

While they were in the pre-fan stage, the nine elite monitors were classified into three categories in terms of their interest in baseball or professional baseball. (a) Three elite monitors didn't have interest in baseball, (b) another three were interested in baseball in general but did not have interest in professional baseball, and (c) the rest had interest in professional baseball but were not interested in purchasing tickets to visit Sapporo dome for watching Fighters' games.

Figure 4 illustrates the cases of the groups (a) and (c). The pre-fans who didn't know baseball well, (a), have developed into either repeaters who enjoy cheering or those who enjoy watching games. The pre-fans in the (c) category have developed into repeaters who enjoy watching games.

\section{Conclusion}

The case study revealed histories of nine elite monitors, which demonstrated how they moved through the fan stages, from the pre-fan stage to the fan stage and ultimately to the loyal-fan stage. We identified three features that motivated participants to advance from the fan stage, and ten features that motivated them to advance from the fan stage to the loyal-fan stage. These features should suggest possible paths that potential loyal fans follow and define possible their needs when they are at the pre-fan stage and those when they are at the fan stage in the future.

\section{Acknowledgements}

Part of this study was supported by the Ministry of Economy, Trade and Industry.

\section{References}

[1] J.S.B.T. Evans and K. Frankish, eds., In Two Minds: Dual Processes and Beyond, Oxford University Press, Oxford, 2009.

[2] D. Kahneman, D. (2003). A perspective on judgment and choice. American Psychologist, 58 (2003), 697-720.

[3] M. Kitajima, H. Tahira, S. Takahashi, and T. Midorikawa, Understanding tourist's in situ behavior: a Cognitive ChronoEthnography study of visitors to a hot spring resort, Journal of Quality Assurance in Hospitality \& Tourism, in press.

[4] M. Kitajima and M. Toyota, Simulating Navigation Behaviour Based on the Architecture Model MHP/RT, Behaviour and Information Technology, in press.

[5] M. Kitajima, M. Nakajima, and M. Toyota, Cognitive ChronoEthnography: A method for studying behavioral selections in daily activities, in: Proceedings of the Human Factors and Ergonomics Society 54th Annual Meeting, 2000, pp. 1732-1736.

[6] E. Someya, M. Kitajima, H. Tahira, and T. Kajikawa, Project B*B: Developmental processes of fan loyalty for the professional baseball team 'The Hokkaido Nippon-Ham Fighters', in: Proceedings of the 2009 North American Society for Sport Management Conference (NASSM 2009), 2009, pp. 364-365. 